Anaesthesist 2018 $\cdot 67: 79-80$

https://doi.org/10.1007/s00101-018-0415-8

Online publiziert: 31. Januar 2018

(c) Springer Medizin Verlag GmbH, ein Teil von Springer Nature 2018

CrossMark

\title{
B. Sinner
}

Klinik für Anaesthesiologie, Universität Regensburg, Regensburg, Deutschland

\section{Onkologie in Anästhesie und operativer Intensivmedizin}

Zahl von Patienten klagt im Verlauf ihrer Erkrankung über Schmerzen und benötigt eine differenzierte Tumorschmerztherapie [2]. Besonders unter Chemotherapie, aber auch bei verdrängendem Tumorwachstum klagen die Patienten über Übelkeit und Erbrechen sowie chronische Müdigkeit oder Gedächtnisstörungen [3]. Ein großer Teil der Patienten mit maligner Erkrankung entwickelt im Verlauf eine Anorexie mit Gewichtsverlust und eingeschränkter körperlicher Belastbarkeit. Dies hat unmittelbare Auswirkungen auf das postoperative Outcome und ist z. B. mit Wundheilungsstörungen assoziiert [4].

Besonders kardiovaskuläre Begleiterscheinungen der Tumorerkrankung und -therapie sind für den Anästhesisten und Intensivmediziner von Bedeutung. Hier lassen sich Herzrhythmusstörungen, eine bestrahlungs- oder antracyclininduzierte Kardiomyopathie oder eine Herzinsuffizienz beobachten $[5,6]$. $\mathrm{Zu}$ den vaskulären Komplikationen bei Tumorpatienten zählen v.a. die tiefe Beinvenenthrombose, die man in $5-10 \%$ der Fälle postoperativ antreffen kann, oder auch die periphere arterielle Verschlusskrankheit [6]. Circa 5-10\% dieser Patienten zeigen pulmonale Reaktionen wie z. B. Strahlenpneumonitis, bevorzugt in Kombination mit Sauerstoff oder Chemotherapie [6].

Für Anästhesisten und Intensivmediziner spielen besonders hämatologische Komplikationen der Tumorerkrankung und -therapie eine große Rolle. Eine Anämie kann Folge einer akuten Blutung aus dem Tumor, einer tumorbedingt verminderten Erythropoetinbildung oder auch der Chemotherapie sein [7]. Besonders im Zusammenhang mit der Chemotherapie werden immer wieder eine Leukound Neutropenie beobachtet, die zu einer verstärken Infektanfälligkeit führen [8]. Eine Thrombopenie kann medikamentös induziert oder Folge einer verstärkten Sequestration von Thrombozyten in der Milz sein.

Nicht selten können bei Patienten mit Malignomen endokrinologische Veränderungen und Störungen des Elektrolythaushalts beobachtet werden. Hierzu zählt die Hyperkalzämie, die bei ca. $10 \%$ der Patienten beobachtet werden kann und gehäuft im Zusammenhang mit Bronchialkarzinomen oder Knochenmetastasen auftreten können [9]. Eine Störung des Wasserhaushalts z.B. im Rahmen einer inadäquaten $\mathrm{ADH}$ (Antidiuretisches Hormon)-Sekretion führt zu einer Hyponatriämie und Wasserretention [9].

\section{Der Krebspatient auf der operativen Intensivstation}

Die steigende Zahl und verbesserte Therapie von Patienten mit Malignomen macht es wahrscheinlich, dass solche Patienten aus unterschiedlichsten Indikationen auf Intensivstationen aufgenommen werden [10]. Die Ursache für die Aufnahme kann dabei ebenfalls tumorassoziiert, therapieassoziiert oder aber völlig unabhängig von der Grunderkrankung sein. Hier setzt der vorliegende Beitrag der Autoren um Herrn Prof. Annecke an [11]. Sie beschreiben detailliert und ausführlich mögliche tumorassoziierte Indikationen und therapieassoziierte Ursachen, die zur Aufnahme auf eine Intensivstation führen können. Dabei legen die Autoren besonderen Wert auf die Darstellung der Wirkungen und Nebenwirkungen der modernen Tumortherapieformen, wie die Beeinflussung der zellulären Imbeeinträchtigt werden kann. Eine große 
munantwort durch neue Medikamente wie monoklonale oder bispezifische Antikörper oder Immun-CheckpointInhibitoren. Kernstück der Arbeit sind Kriterien, die für die Entscheidung zur Aufnahme von Krebspatienten auf Intensivstation in Betracht gezogen werden sollten und für den weiteren Krankheitsverlauf entscheidend sein können [11].

Inwiefern durch anästhesiologische und intensivmedizinische Maßnahmen wie die Anlage von Regionalanästhesieverfahren oder die Verwendung bestimmter Anästhetika ein positiver Einfluss auf den Tumorprogress und die Rezidiv- bzw. Metastasierungsrate genommen werden kann, ist aktuell Gegenstand intensiver Forschungsarbeit. Nach der derzeitigen Studienlage kann für ein spezifisches perioperatives Vorgehen keine Empfehlung ausgesprochen werden. Maßnahmen zur Vermeidung von Kontamination, Hypothermie, Blutverlust und Transfusion sind sinnvoll, um die perioperative Morbidität und Letalität onkologischer Patienten zu reduzieren.

\section{B. huner}

B. Sinner

\section{Korrespondenzadresse}

\section{PD Dr. B. Sinner, D.E.A.A}

Klinik für Anaesthesiologie, Universität

Regensburg

Franz-Josef-Strauss Allee 11, 93053 Regens-

burg, Deutschland

barbara.sinner@klinik.uni-regensburg.de

Interessenkonflikt. B. Sinner gibt an, dass kein Interessenkonflikt besteht.

\section{Literatur}

1. RKI (2017) Krebs in Deutschland für 2013/2014. Zentrum für Krebsregisterdaten. Robert Koch Institut, Berlin

2. Grossman SA, Sheidler VR (2000) Cancer pain. In: Abeloff MD, Armitage JO, Lichter AS (Hrsg) Clinical Oncology, 2. Aufl. Churchill Livingstone, New York, S539-555

3. Kiyomiya K, Matsuo S, Kurebe M (2001) Differences in intracellular sites of action of adriamycin in neoplastic and normal differentiated cells. Cancer Chemother Pharmacol 47:51-56

4. Bozzetti F, Bozetti F, Migliavacca S, Scotti A et al (1982) Impact of cancer, type, site, stage and treatment on the nutritional status of patients. Ann Surg 196:170-179

5. Payne WG, Naidu DK, Wheeler CK et al (2008) Wound healing in patients with cancer. Eplasty 8:e9

6. Chang HM, Moudgil R, Scarabelli T, Okwuosa TM, Yeh ETH (2017) Cardiovascular complications of cancer therapy: best practices in diagnosis, prevention, and management: part 1. J Am Coll Cardiol 70:2536-2551

7. Chang HM, Okwuosa TM, Scarabelli T, Moudgil R, Yeh ETH (2017) Cardiovascular complications of cancer therapy: best practices in diagnosis, prevention, and management: part 2. J Am Coll Cardiol 70:2552-2565

8. Gilreath JA, Stenehjem DD, Rodgers GM (2014) Diagnosis and treatment of cancer-related anemia. Am J Hematol 89:203-212

9. Dimitriadis GK, Angelousi A, Weickert MO et al (2017) Paraneoplastic endocrine syndromes. Endocr Relat Cancer 24:R173-R190

10. Lambertz R, Drinhaus H, Schedler D et al (2016) Perioperative management of transthoracic oesophagectomies: Fundamentals of interdisciplinary care and new approaches to accelerated recovery after surgery. Anaesthesist 65:458-466

11. Annecke AT, Hohn A, Böll B, Kochanek M (2018) Krebspatienten in der operativen Intensivmedizin. Anästhesist. https://doi.org/10.1007/s00101018-0406-9
Forschungsgruppe des European Resuscitation Council (ERC) erhält lan G. Jacobs Award der American Heart Association (AHA) für internationale Forschungskooperation

Der lan G. Jacobs Award ehrt internationale Forschungskollaborationen im Bereich der erweiterten Reanimationsforschung. Auf dem Resuscitation Science Symposium der American Heart Association (AHA) ist die europäische Forschungsgruppe des European Resuscitation Council (ERC) um Prof. Dr. Bernd Böttiger, Uniklinik Köln, ausgezeichnet worden. Die Gruppe erhielt die Auszeichnung für ihre Forschungsaktivitäten im Rahmen des Europäischen Reanimationsregisters.

Die Wissenschaftler erhoben In der bisher größten europäischen Registerstudie „EuReCa ONE“ im Jahr 2014 erstmals außerklinische Reanimationsergebnisse aus 27 europäischen Ländern gleichzeitig, um sie hinsichtlich Inzidenz, Versorgungsprozessen und Ergebnissen bzw. Outcome zu vergleichen. Das europäische Register wurde im Jahr 2007 unter dem Dach des ERC mit Unterstützung von Prof. Dr. Bernd Böttiger, Uniklinik Köln, als damaligem Präsident, gegründet.

Der lan G. Jacobs Award wird seit 2010 an internationale Forschungsgruppen verliehen, die sich in besonderer Weise im Bereich der erweiterten Reanimationsversorgung engagieren und hierbei richtungsweisende Ergebnisse mit hoher wissenschaftlicher Relevanz für die Versorgung von Menschen mit Herzkreislaufstillstand hervorbringen. Der Award wird von der AHA zu Ehren von Professor Ian George Jacobs, PhD, OStJ, FERC, FAHA verliehen, der sich unermüdlich dafür eingesetzt hat, Mediziner und Wissenschaftler unterschiedlichster Disziplinen weltweit zusammen zu bringen, um die globale Versorgung von Menschen mit plötzlichem Herzkreislaufstillstand zu verbessern.

Mehr Informationen unter

https://www.eureca-one.eu

Quelle: Uniklinik Köln, www.uk-koeln.de 